\title{
NOTES AND COMMENTS
}

\section{HETEROZYGOUS ADVANTAGE AS A LIKELY GENERAL BASIS FOR ENZYME POLYMORPHISMS}

\author{
J. R. S. FINCHAM \\ Department of Genetics, University of Leeds, Yorks., England
}

Received 7.xii.71

THE development of methods for resolving variant proteins by electrophoresis in starch or polyacrylamide gels has led to the recognition, in populations of a wide variety of organisms, of polymorphisms with respect to a high proportion of the enzymes amenable to study. Much of the information available has been reviewed by Manwell and Baker (1970). According to one school of thought such widespread polymorphism can be accounted for by the occurrence of mutations of neutral survival value (Kimura and Ohta, 1971), though the likelihood of many mutations being truly neutral is still disputed (Clark, 1970; Richmond, 1970). Alternative selectionist interpretations of the phenomenon can invoke frequencydependent selection, periodic or disruptive selection (different genotypes selected for at different times or in different places in a variable environment) or heterozygous advantage. The idea of the rather general superiority of heterozygotes is one which has long had a considerable following, but a plausible rationalisation of such an effect has been lacking and belief in it has often seemed to rest more on faith than on reason. It is the purpose of this communication to argue that, in an outbreeding species, the widespread occurrence of heterozygous advantage is very much to be expected and may well provide a sufficient though, of course, not necessarily the only reason for the frequent occurrence of enzyme polymorphisms.

In considering the effects of heterozygosity, the classical notions of dominance and recessiveness may have conditioned our thinking unduly. While the common existence of incomplete dominance is universally recognised, there is still a widespread feeling that it is, if not exceptional, still far from being the rule. This feeling has some justification at the level of morphology, but when enzyme phenotypes are considered, intermediacy of heterozygotes is almost universal. In cases of alleles determining normal versus low or zero amounts of a specific enzyme one almost always finds intermediate enzyme levels in diploid heterozygotes or haploid fungal heterokaryons. Alleles determining qualitatively (e.g. electrophoretically) distinct varieties of an enzyme can, furthermore, frequently be shown to interact in heterozygotes or heterokaryons to form hybrid oligomeric enzyme forms with intermediate properties. Admittedly, this lack of dominance at the biochemical level is often not manifest at the level of morphology; organisms have complex homoeostatic controls which tend to minimise any overt disruption of normal metabolism or development by moderate variations in enzyme activities. Nevertheless, it would be unwarranted to conclude that the underlying biochemical variations are without significance for fitness. Different genotypes may achieve a superficially standard phenotype but still differ markedly in the extent to which their metabolism was 
stressed in the process; under less favourable conditions those with weaker or less well controlled enzymic equipment could have a reduced chance of survival.

There are at least two ways in which the heterozygous combination of co-dominant alleles could confer a selective advantage. First, and most simply, the new allele could, in combination with the old, produce a quantity of enzyme activity closer to the optimum in the prevailing environment than had been given by the progenitor homozygote. In such a case the effect of the new allele when homozygous might very well be too extreme. A purely quantitative effect of this kind could, of course, operate without any distinctive heterozygote protein product being formed-the advantage would rest merely on the additive action of the two alleles.

Rather more interesting is the possibility that the interaction of an old with a new allele could give a qualitatively distinct enzyme with uniquely advantageous properties. Such a situation is easiest to visualise in the case of an enzyme with allosteric properties; that is, with an interconversion between active and inactive conformations controlled by the concentration of a small-molecular allosteric effector. It is characteristic of allosteric enzymes that the conformational transition occurs over a rather narrow range of effector concentration, this range reflecting, presumably, the critical physiological or nutritional circumstances under which it is to the organism's advantage to switch the enzyme activity on or off. It is easy to see that with changed nutritional conditions such as might, for example, accompany a climatic change or a colonisation of new territory, the setting of the chemostat might need to be adjusted-that is to say, it might well be a selective advantage to modify the allosteric properties of an enzyme so that it made its conformational transition at a somewhat higher or lower effector concentration than before.

We know, in fact, that the allosteric properties of an oligomeric allosteric enzyme may be quite strikingly modified in a heterozygote or heterokaryon between the original wild allele and a mutant derivative through the formation of heteroligomeric enzyme molecules. Two examples of such an effect may be cited.

Among the mutants of Neurospora crassa with abnormal NADP-linked glutamate dehydrogenase $(\mathrm{GDH})$ are several which produce forms of the enzyme with altered responses to allosteric effectors. The wild-type enzyme is inactive in the absence of effectors at $p \mathrm{H} 7.0$ but can be activated at this $p \mathrm{H}$ by the addition of either glutamate or succinate (West et al., 1967). Above $p \mathrm{H} 8.0$ the enzyme is virtually fully active even in the absence of the effectors. One mutant form of the enzyme $\left(a m_{3}\right)$ is inactive even at $p \mathrm{H} 8.5$ in the absence of effectors but can be activated by relatively high concentrations of glutamate or succinate (Fincham, 1962). This mutant enzyme is apparently inactive under all normal intracellular conditions. Heterokaryons formed between wild-type and $a m_{3}$ form a proportion of hybrid oligomeric enzyme molecules with intermediate properties (Coddington, 1966). The figure shows that the enzyme extracted from the $a m++a m_{3}$ heterokaryon is heterogeneous (there being the possibility of several different hybrids of different compositions as well as of a proportion of homooligomers), and one fraction behaves rather like pure wild and another like pure $a m_{3}$. A proportion of the activity, however, responds to a change in succinate concentration over a range $(7.5$ to $15 \mathrm{mM})$ in which the am+ enzyme is 
fully active and the $a m_{3}$ enzyme quite inactive. The physiological significance of the response of Neurospora GDH to glutamate or succinate is unclear, but this example can at least serve as a model of the way in which an allosteric response can be modulated by the incorporation of mutant monomers in a hybrid oligomeric enzyme.

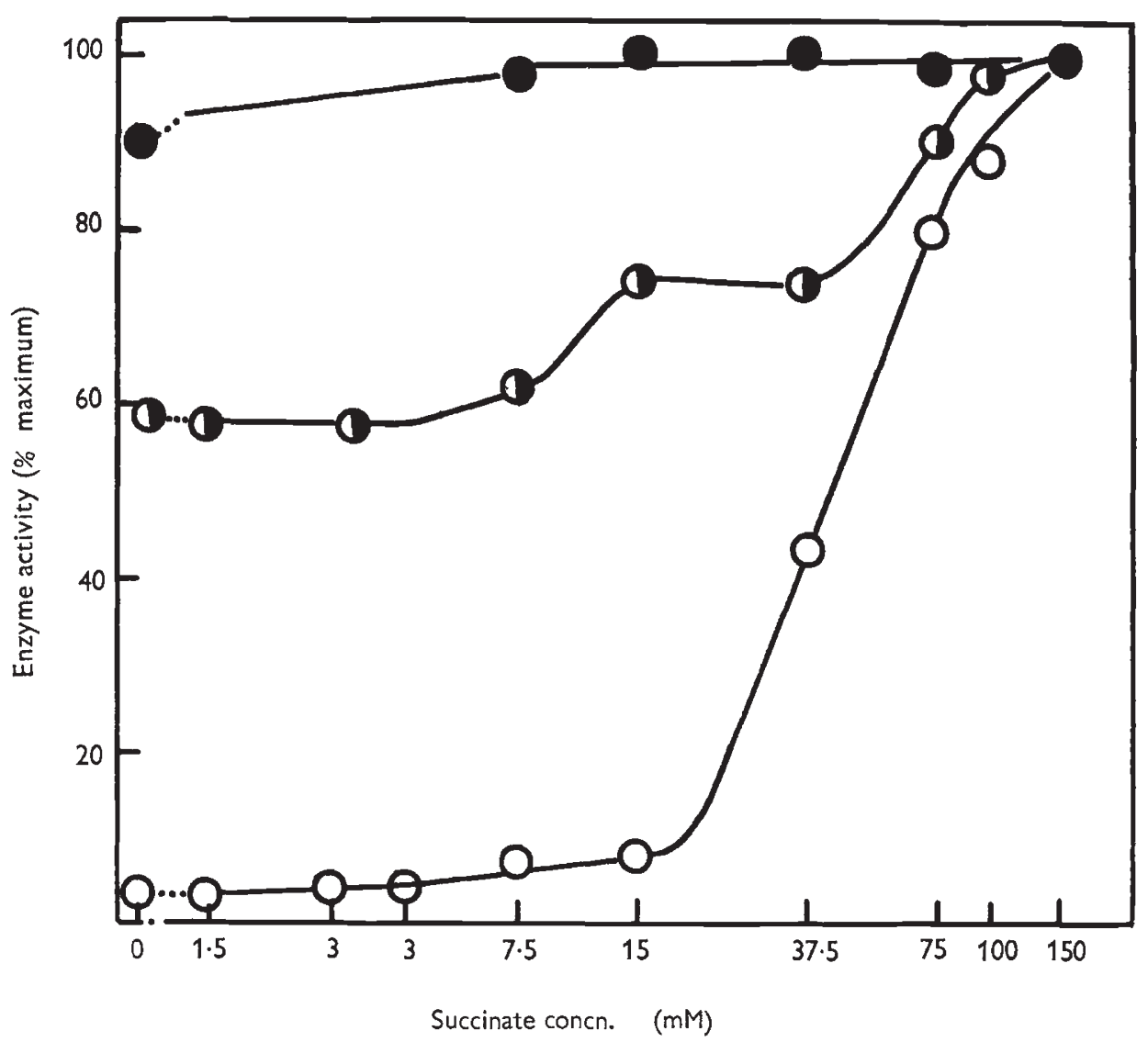

Fig. 1.-The different responses of glutamate dehydrogenase from Neurospora wild type (filled circles), $a m_{3}$ mutant (open circles) and wild $+a m_{3}$ heterokaryon (half-filled circles) to succinate as an activator. Mycelial extracts were incubated at $35^{\circ}$ in Trishydrochloride buffer at $p \mathrm{H} 8 \cdot 5$, and the indicated concentrations of disodium succinate, in volumes of $0.2 \mathrm{ml}$. After 5 minutes of incubation the whole $0.2 \mathrm{ml}$. was added to $2.8 \mathrm{ml}$. of a Tris-glutamate-NADP mixture at $35^{\circ}$ (assay system " $\mathrm{C}$ " of Fincham, 1962 ) and the enzyme reaction was followed by recording increase of optical density at $340 \mathrm{~nm}$. The initial reaction rates were used to determine the percentage of full enzyme activity at the time of addition to the assay system. The scale of succinate concentration is logarithmic for convenience in plotting.

A second example is provided by the studies of Zimmerman and Gundelach (1969) on threonine dehydratase in yeast. These workers showed that a mutant allele which, by itself, produced an almost inactive enzyme variety, formed in heterozygous diploid combination with the wild-type allele a hybrid enzyme with reduced sensitivity to feedback inhibition by isoleucine. 
In each of the cases just considered the mutation has a rather extreme effect and the mutant homokaryon or homozygote would hardly be viable at all in a natural population. We can easily imagine less drastic modifications, less likely to be picked up in laboratory mutant hunts but more important in nature, which would exercise more subtle effects in heterozygotes and still lead to significantly reduced viability when homozygous.

Perhaps it should be stressed that the argument presented here does not lead to the conclusion that there is any advantage in heterozygosity per se. In seeking the optimum level of activity, or control of allosteric response, of an enzyme, a diploid organism has a wide range of possibilities, all attainable through single mutational steps. Each of $n$ possible mutant alleles could in principle be obtained either homozygous or in heterozygous combination with the progenitor allele. Each of these $2 n$ faccihilities may produce a distinct enzyme phenotype and the chance of finding a better adapted enzyme may be expected, a priori, to be as good among the homozygotes as among the heterozygotes. But whereas in a habitually self-fertilising or closely inbreeding species the mutant homozygote will be available very soon and the heterozygote lost by segregation, in an outbreeding species with a large effective population size any new allele will remain almost entirely in heterozygotes for a long time. Thus in the short or medium term inbreeding and outbreeding species will tend to "choose" alternative sets of options - that is, mutations showing homozygous and heterozygous advantage respectively. It is indeed true that a mutant allele which brings some advantage when heterozygous and more when homozygous will in the long run overtake and replace any other allele which brings maximum advantage when heterozygous, but is less advantageous in the homozygote. Mandel (1959) and $\mathrm{Li}$ (1967) have considered such situations. There are, however, two reasons why this consideration does not argue at all strongly against the general explanation of enzymic polymorphism which I am advocating. Firstly, there is no general reason to expect that because a mutation confers some advantage when heterozygous, it will be even more beneficial when homozygous. Even if the effect in the heterozygote is merely a quantitative one, supplementing or diluting the activity of the progenitor allele without changing the kind of activity, its effect when homozygous will as likely as not be to overshoot the optimum. If, on the other hand, the heterozygous advantage is the result of an interaction between progenitor and mutant allelic products in a hybrid protein, then the properties of the homozygous mutant protein could be radically new and it will be gratuitous good fortune if they turn out to be more advantageous than those of the hybrid. Secondly, even if such alleles (i.e. good in heterozygotes-even better in homozygotes) are available, it may take them so long to become fixed that a polymorphic situation, based on alleles which bring a quick maximum benefit in heterozygotes, may persist for a long time in a population under selection in a new environment. The polymorphisms which we see in natural populations need not represent true equilibria; indeed most land environments may not be sufficiently stable for long enough for true equilibria ever to be attained.

I conclude that it is not at all surprising that enzyme polymorphisms are so frequent in natural outbreeding populations. Had they not already been discovered our knowledge of the effects of mutations on enzyme activities should have enabled us to predict them. It must be admitted, however, that direct evidence is as yet rather scant and conflicting. Thus in Drosophila, 
one enzyme polymorphism has resisted attempts to show that it effects fitness in any way (Yamazaki, 1971) while in other cases (e.g. Richmond and Powell, 1970) the data do favour heterosis associated with a hybrid enzyme. What needs to be demonstrated, of course, is that the nature of the presumed advantage attaching to hybrid enzymes in naturally occurring polymorphisms is of the kind suggested here. Information on possible allosteric properties of polymorphic enzymes would be especially interesting.

\section{SUMMARY}

1. It is argued that, in outbreeding diploid species, selection for improved enzyme function is likely to lead to the establishment of new alleles which are advantageous in heterozygotes rather than homozygotes, and that the common enzyme polymorphism shown by natural populations may be explained on this basis.

2. The possible nature of heterozygous advantage is discussed and it is suggested that it may have either a quantitative or a qualitative basis. The distinctive allosteric properties of hybrid enzymes may be important in this connection.

Acknowledgments. -I thank Drs B. Shorrocks and M. d'A. Crawfurd for comments on a draft of this communication.

\section{REFERENGES}

CLARK, B. 1970. Darwinian evolution of proteins. Science, 169, 1009.

CoDdington, A. 1966. Hybridization in vitro between wild-type and mutant forms of glutamate dehydrogenase from Neurospora crassa. Biochem. $\mathcal{7} ., 99,9 c$.

FINCHAM, J. R. s. 1962 . Genetically determined multiple forms of glutamic dehydrogenase in Neurospora crassa. J. mol. Biol., 4, 257.

KIMURA, M., AND OHTA, T. 1971. Protein polymorphism as a phase of molecular evolution. Nature, 229, 467.

L1, c. c. 1967. Genetic equilibrium under selection. Biometrics, 23, 397.

MANDEL, S. P. H. 1959. The stability of a multiple allelic system. Heredity, 13, 289.

MANWEll, C., AND BAKer, C. M. A. 1970. Molecular Biology and the Origin of the Species. Pp. 394. Sidgwick \& Jackson.

RICHMOND, R. C. 1970. Non-Darwinian evolution: a critique. Nature, 225, 1025.

RICHMOND, R. C., AND POWELL, J. R. 1970. Evidence of heterosis associated with an enzyme locus in a natural population of Drosophila. Proc, nat. Acad. Sci., Wash., 67, 1264.

WEST, D. J., TUVESON, R. W., BARRATT, R. W., AND FINCHAM, J. R. s. 1967. Allosteric effects in nicotinamide adenine dinucleotide phosphate-specific glutamate dehydrogenase from Neurospora. J. biol. Chem., 242, 2134.

xAMAZAKI, T. 1971. Measurement of fitness at the esterase-5 locus in Drosophila pseudoobscura. Genetics, 67, 579.

ZIMMERMAN, F. K., AND GUNDELACH, E. 1969. Intragenic complementation, hybrid enzyme formation and dominance in diploid cells of Saccharomyces cerevisae. Molec. Gen. Genetics, 103,348 . 